\title{
Fleischner recommendations for the management of subsolid pulmonary nodules: high awareness but limited conformance - a survey study
}

\author{
Onno M. Mets ${ }^{1}$ - Pim A. de Jong ${ }^{1}$ - Kaman Chung ${ }^{2}$ - Jan-Willem J. Lammers ${ }^{3}$. \\ Bram van Ginneken ${ }^{2}$ Cornelia M. Schaefer-Prokop ${ }^{2,4}$
}

Received: 6 August 2015 /Revised: 29 December 2015 / Accepted: 25 January 2016 / Published online: 5 March 2016

(C) The Author(s) 2016. This article is published with open access at Springerlink.com

\begin{abstract}
Objectives The aim of this study was to assess awareness and conformance to the Fleischner society recommendations for the management of subsolid pulmonary nodules (SSN) in clinical practice.

Methods An online questionnaire with four imaging cases was sent to 1579 associates from the European Respiratory Society and 757 from the European Society of Thoracic Imaging. Each respondent was asked to choose from several options which one they thought was the indicated management for the nodule presented. Awareness and conformance to the Fleischner recommendations (FR) were assessed and correlated to respondents characteristics.

Results In total, 119 radiologists (response rate $16.0 \%$ ) and 243 pulmonologists (response rate $16.5 \%$ ) were included. Awareness of the FR was higher in radiologists than in pulmonologists ( $93 \%$ vs. $70 \%, \mathrm{p}<0.001$ ), as was implementation in daily practice (66\% vs. $47 \%, \mathrm{p}<0.001)$. Radiologists conformed to FR in rates of $31,69,68$, and
\end{abstract}

Electronic supplementary material The online version of this article (doi:10.1007/s00330-016-4249-y) contains supplementary material, which is available to authorized users.

Onno M. Mets

o.m.mets@umcutrecht.nl

1 Department of Radiology, University Medical Center Utrecht, Utrecht, The Netherlands

2 Diagnostic Imaging Analysis Group, Radboud University Nijmegen Medical Centre, Nijmegen, The Netherlands

3 Department of Pulmonology, University Medical Center Utrecht, Utrecht, The Netherlands

4 Department of Radiology, Meander Medical Center, Amersfoort, The Netherlands

$82 \%$, and pulmonologists in $12,43,70$, and $75 \%$ for cases 1 to 4 , respectively. Overmanagement was common. Conformance in SSN management was associated with awareness, working in an academic practice, larger practice size, teaching residents, and higher SSN exposure.

Conclusions Although awareness of the Fleischner recommendations for SSN management is widespread, management choices in clinical practice show large heterogeneity.

Key points

- Guideline awareness among clinicians is widespread, but conformance shows large heterogeneity.

- Awareness and conformance is significantly higher among radiologists than pulmonologists.

- Overmanagement is common, which may lead to avoidable financial and physical burden.

Keywords Solitary pulmonary nodule $\cdot$ Adenocarcinoma . Questionnaires · Guideline adherence · Evidence based practice

\begin{abstract}
Abbreviations
SSN Subsolid nodule

FR Fleischner recommendations (for the management of subsolid pulmonary nodules)
\end{abstract}

\section{Introduction}

Pulmonary nodules represent one of the most frequent incidental findings. Subsolid pulmonary nodules (SSNs) form a distinct subgroup of lung nodules, with different morphology and clinical behaviour. Apart from density, the most striking differences are the higher malignancy rate [1] and slower growth rate [2] compared to solid pulmonary nodules. These 
characteristics lead to an excellent prognosis when treated early [3-5], however, they also pose the risk of overdiagnosis and overtreatment $[6,7]$. A growing understanding of the meaning of the SSN entity and its stepwise progression in the adenomateous spectrum has considerably increased SSN awareness over the last several years. Subsequently, both radiologists and clinicians are increasingly confronted with the dilemma of choosing the most adequate interpretation and management.

In 2013 the Fleischner Society published recommendations for the management of SSNs in daily practice, based on careful review of the available scientific evidence and expert opinion [8]. The need for such a directive is apparent from the fact that the report is listed in the online top ten most-viewed articles in RADIOLOGY [9]. Such a fundamental document may help to align management in clinical practice, increase health care quality, and decrease unnecessary procedures. However, to achieve this, it is important that both radiologists and pulmonologists are not only familiar with the Fleischner recommendations (FR), but moreover, act accordingly. Two years after publication it is unknown to which degree the FR are known and indeed applied in daily practice. Therefore, the purpose of this study was to assess awareness and quantify conformance to FR among radiologists and pulmonologists in daily practice.

\section{Materials and methods}

Approval by the institutional ethical review board of the University Medical Center Utrecht, was waived due to the study design.

\section{Respondents}

An invitation to complete an online questionnaire was sent out through the European Society of Thoracic Imaging (ESTI) and the European Respiratory Society (ERS). The ESTI society sent a mailing to 757 unique recipients that were either ESTI members or had visited the annual ESTI conference of 2014. The ERS selected 1579 unique subjects in five relevant scientific groups (i.e. imaging, interventional pulmonology, diffuse parenchymal disease, lung cancer, and pleural and mediastinal malignancies). The survey was open for 4 weeks. A reminder was sent 1 week before closure.

\section{Online survey}

The questionnaire presented four cases with either a new or a persistent pulmonary nodule. Each case was presented with short clinical information on sex, age, and nodule persistency. The nodules were displayed using both a single-axial computed tomography (CT) slice centred at the lesion, as well as an animated figure that automatically and repeatedly scrolled through the abnormality, providing the full volumetric information in an axial projection. CT images had been acquired using a low-dose protocol (120 or $140 \mathrm{kV}$ at 30 $\mathrm{mAs}$ ) with a smooth reconstruction kernel (C- or B-filter, Philips Healthcare, Best, The Netherlands). Diameters of the total lesion and the solid component (in $\mathrm{mm}$ ) were determined manually in the transverse plane using the lung setting (L-600, W1600). Case 1 showed a persistent, pure ground-glass SSN $(15 \times 14 \mathrm{~mm})$, case 2 presented a new part-solid SSN with a small solid component (total lesion size 20x16 mm, solid component size $3 \times 4 \mathrm{~mm}$ ), case 3 showed a persistent partsolid SSN with a large solid component (total lesion size $25 \times 25 \mathrm{~mm}$, solid component size $12 \times 8 \mathrm{~mm}$ ), and case 4 was a solid triangular nodule with perifissural location near a vein (10x5 mm), illustrating the typical morphology of a benign intrapulmonary lymph node [10]. Figure 1 presents all four imaging cases. The animated figures can be found in the online supplement.

First, the recipients were asked whether they thought the presented nodule concerned a subsolid nodule, and if so, to select what they thought was best medical practise from several management options: (a) nothing; (b) 4-week follow-up; (c) 3-month follow-up; (d) 6month follow-up; (e) 24-month follow-up; (f) serial follow-up in 12, 24, and 36 months; (g) additional diagnostics (PET-CT, biopsy, etc.); (h) resection of (an almost) certain malignancy; or (i) other. Respondents were limited to providing a single best answer.

Apart from the four imaging cases, the questionnaire also contained a variety of questions focussed on the background and experience of the respondent (e.g. years of experience, type of hospital, practice size). Regarding this information, two slightly different questionnaires were designed for radiologists and pulmonologists. Both questionnaires can be found in the online supplement.

\section{Analysis}

For each case presented, the best management option according to the FR was determined in consensus by three of the authors (OMM, PAJ, and CSP, with 5, $>10$, and $>20$ years of experience in thoracic radiology, respectively). This was used as the reference standard. According to the FR the persistent pure SSN in case 1 required serial follow-up at 12, 24, and 36 months. The newly detected part-solid SSN in case 2 required a 3month follow-up to evaluate persistence. The persistent part-solid SSN in case 3 should be considered a malignancy and required additional action (either resection or PET-CT/biopsy; both answers were considered conform the FR), while the perifissural nodule (PFN) in case 4 
Fig. 1 Imaging cases used in the questionnaire. Clockwise from the upper-left panel, the figure shows a persistent pure groundglass nodule (case 1), a new partsolid lesion with a small solid component (case 2), a persistent part-solid lesion with larger solid component (case 3), and a triangular solid nodule (case 4)
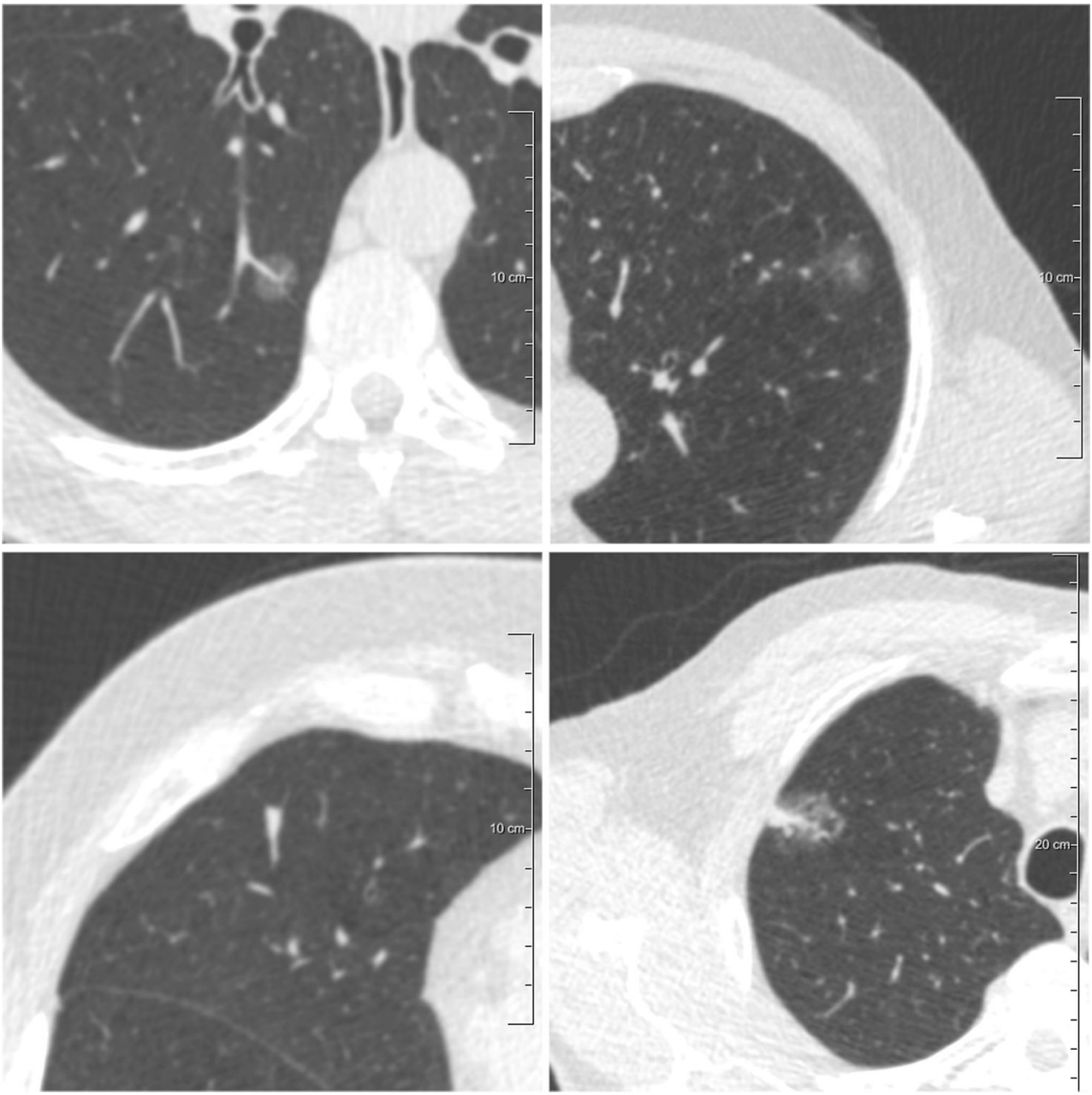

did not require any follow-up, and in fact did not represent the type of nodule to which the Fleischner recommendations for SSN management apply.

Respondents' answers were compared to the abovementioned reference standard, and recommended management was divided into three categories: (1) conforms to the FR, (2) undermanagement (defined as failure to obtain follow-up at all, failure to obtain the recommended follow-up in time, or failure to perform resection/PET/biopsy in a highly suspicious lesion), or (3) overmanagement (defined as shorter/more frequent follow-up than recommended, or use of additional imaging modalities/invasive techniques considered not indicated). The respondents' management choices were evaluated in relation to their clinical background and experience.

Statistical analyses were performed using SPSS v20.0 for Windows (SPSS, Chicago, Illinois, USA). Comparison between groups (proportions) were performed using chisquared testing or the Fischer exact test, as appropriate. A pvalue below 0.05 was considered to represent a significant difference.

\section{Results}

\section{Respondents}

In total, 121 completed questionnaires were received through ESTI, as were 260 through ERS (response rate $16.0 \%$ and $16.5 \%$, respectively). Seventeen respondents were excluded due to other job descriptions or incorrect completion of the questionnaire (five radiologists filled in the pulmonology survey and one pulmonologist filled in the radiology survey). Also, two duplicate respondents were excluded. This yielded a total of 362 completed questionnaires by respondents originating from 60 countries (255 European $(71 \%)$, five missing). Figure 2 shows the flow chart of the respondent inclusion procedure. Table 1 shows the demographic data of the included respondents.

\section{Awareness and application in daily practice}

Radiologists reported being aware of the Fleischner recommendation for SSN management in $93 \%$ of the 


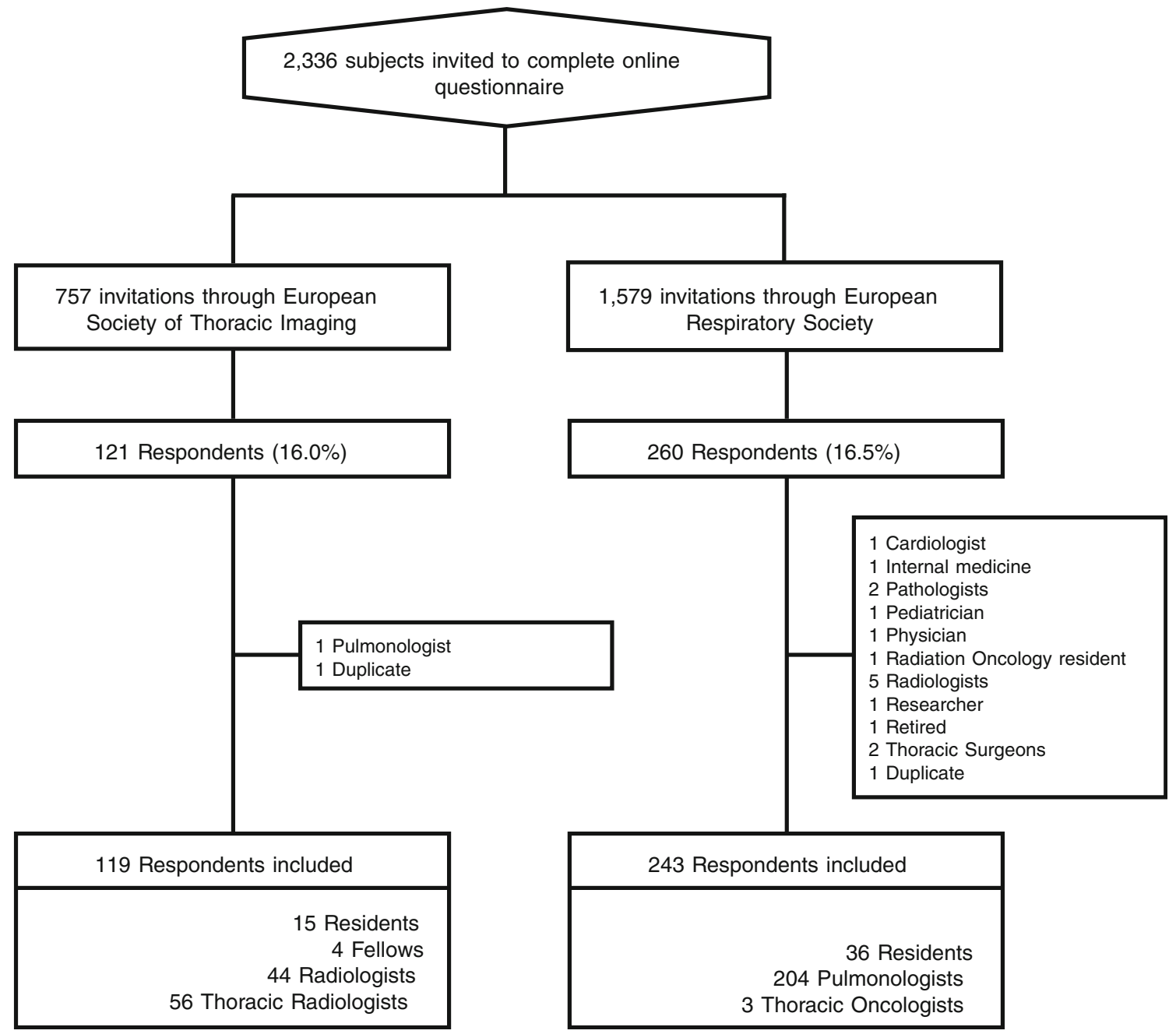

Fig. 2 Flow chart of respondent inclusion

responses, compared to $70 \%$ of the pulmonologists $(\mathrm{p}<0.001)$. Radiologists reported to apply them in daily practice at a rate of $66 \%(78 / 119)$, while $17 \%(20 /$ $119)$ used a locally developed protocol and $10 \%$ (12/ $119)$ reported no consistent way of handling SSNs. Only a small proportion of radiologists $(3 \%, 5 / 119)$ did not (or only did so in a minority of cases) include recommendations for nodule management in their report. Overall, $94 \%$ of the radiologists reported moderate to above-average confidence in SSN management (111/ 119). Pulmonologists adhered to the FR at a rate of $47 \%(113 / 243)$, while $26 \%$ used a locally developed protocol (62/243), and $18 \%$ reported no consistent way of handling SSNs (43/243). In the case of a recommendation in the report, $47 \%(115 / 243)$ replied to follow this advice most of the times/always, while $10 \%(24 /$ $243)$ ignored it most of the times. Only $38 \%(92 / 243)$ of the pulmonologists experienced moderate to aboveaverage confidence in managing SSNs $(p<0.001$ compared to radiologists).
Nodule classification and management recommendations

Tables 2 and 3 outline the chosen management strategies per case, showing that when radiologists rated the nodule as an SSN they recommended a strategy conforming to the FR in 31,69 , and $69 \%$ of responses for cases 1 to 3 , respectively. The corresponding percentages for pulmonologists were 12 , 43 , and $70 \%$, respectively. Radiologists misclassified the lesions in 13,18 , and $13 \%$ of cases, while pulmonologists were incorrect 8,23 , and $26 \%$ of the time in cases 1 to 3, respectively. The solid perifissural nodule was called an SSN by $4 \%$ $(5 / 119)$ of the radiologists and $14 \%(33 / 243)$ of the pulmonologists.

For the four presented nodules, overmanagement was chosen in $18-58 \%$ of the cases by radiologists and $25-83 \%$ by pulmonologists. This happened most in the small, pure ground-glass nodule (case 1). The persistent subsolid lesion with a solid component exceeding $5 \mathrm{~mm}$ (case 3 ) triggered an undermanagement in 33 and $54 \%$ of the radiologists and pulmonologists, respectively. 
Table 1 Demographics of included respondents

\begin{tabular}{|c|c|c|c|}
\hline ESTI respondents $(\mathrm{N}=119)$ & & ERS respondents $(\mathrm{N}=243)$ & \\
\hline Job Title & & Job Title & \\
\hline Radiology resident & $15(13 \%)$ & Pulmonology resident & $36(15 \%)$ \\
\hline (Cardio) thoracic fellow & $4(3 \%)$ & Pulmonologist & $204(84 \%)$ \\
\hline Radiologist & $44(37 \%)$ & Thoracic oncologists & $3(1 \%)$ \\
\hline Thoracic radiologist & $56(47 \%)$ & & \\
\hline Years of experience & & Years of experience & \\
\hline$<5$ years & $21(18 \%)$ & $<5$ years & $68(28 \%)$ \\
\hline 5 to 9 years & $23(19 \%)$ & 5 to 9 years & $43(18 \%)$ \\
\hline 10 to 19 years & $46(39 \%)$ & 10 to 19 years & $63(26 \%)$ \\
\hline 20 years or more & $29(24 \%)$ & 20 years or more & $69(28 \%)$ \\
\hline Type of hospital & & Type of hospital & \\
\hline University/Academic & $67(56 \%)$ & University/Academic & $155(64 \%)$ \\
\hline General & $43(36 \%)$ & General & $55(23 \%)$ \\
\hline Private clinic & $5(4 \%)$ & Private clinic & $19(8 \%)$ \\
\hline Specialized care & $4(3 \%)$ & Specialized care & $14(6 \%)$ \\
\hline Teaching residents & & Teaching residents & \\
\hline Yes & $102(86 \%)$ & Yes & $182(76 \%)$ \\
\hline No & $17(14 \%)$ & No & $61(25 \%)$ \\
\hline \multicolumn{4}{|l|}{ No. of chest CT reports $\mathrm{p} / \mathrm{wk}$} \\
\hline 0 & $2(2 \%)$ & $\mathrm{N} / \mathrm{A}$ & \\
\hline 1 to 49 & $74(62 \%)$ & N/A & \\
\hline 50 to 99 & $30(25 \%)$ & $\mathrm{N} / \mathrm{A}$ & \\
\hline 100 or more & $13(11 \%)$ & N/A & \\
\hline No. of SSN encountered $\mathrm{p} / \mathrm{yr}$ & & No. of SSN encountered $\mathrm{p} / \mathrm{yr}$ & \\
\hline 1 or less & $4(3 \%)$ & 1 or less & $15(6 \%)$ \\
\hline 2 to 10 & $16(13 \%)$ & 2 to 10 & $98(40 \%)$ \\
\hline 11 to 20 & $30(25 \%)$ & 11 to 20 & $54(22 \%)$ \\
\hline 21 or more & $69(58 \%)$ & 21 or more & $76(31 \%)$ \\
\hline
\end{tabular}

ESTI European Society of Thoracic Imaging; ERS European Respiratory Society; SSN Subsolid nodule
Radiologists showed conformance in zero to all four cases in $5,15,36,28$, and $16 \%$ of responses, respectively. For pulmonologists this was $7,31,46,15$, and $2 \%$, respectively.

\section{Correlation between respondents' characteristics and their awareness and conformance}

As shown in Table 4, significantly higher awareness was seen in more experienced radiologists ( $\geq 5$ year experience), and in radiologists and pulmonologists with more SSN exposure (>10 SSNs p/year). Awareness of the FR among the different categories of respondents is shown fully in Supplemental Table S1.

On a per-case basis, higher rates of conformance were seen in all four cases for those who were aware of the FR, worked in a larger practice ( $\geq 10$ specialists), and encountered more SSNs ( $>10 \mathrm{p} /$ year). However, differences did not reach significance in all cases. Awareness among pulmonologists also led to higher conformance in all cases, but conformance was more variable. Overall analysis showed that highly conformant radiologists (defined as: three or all four cases conform to the FR) were significantly more often aware of the FR $(\mathrm{p}<0.01)$, worked in an academic centre $(\mathrm{p}<0.01)$, taught residents more often $(\mathrm{p}=0.02)$, worked in a practise with $\geq 10$ specialists $(p<0.01)$, and encountered $>10$ SSNs $p / y e a r(p<0.01)$. High conformance in pulmonologists was only associated with FR awareness $(p=0.02)$ and $\geq 10$ year experience $(p=0.02)$. Figure 3 graphically summarises the abovementioned results.

\section{Measurement of nodule dimensions}

A minority of radiologists indicated that they obtained measurements using volumetric software $(8 \%, 9 / 119)$. Of the radiologists using calipers, only $9 \%(10 / 110)$ reported obtaining the average of two maximal 
Table 2 Chosen management strategies per case by radiologists or radiology residents

\begin{tabular}{|c|c|c|c|c|c|c|c|c|}
\hline & \multicolumn{2}{|l|}{ Case 1} & \multicolumn{2}{|l|}{ Case 2} & \multicolumn{2}{|l|}{ Case 3} & \multicolumn{2}{|l|}{ Case 4} \\
\hline & SSN & No SSN & SSN & No SSN & SSN & No SSN & SSN & No SSN \\
\hline Management strategy & $103^{*}$ & 16 & $97^{*}$ & 22 & $105^{*}$ & 14 & 5 & $114^{*}$ \\
\hline Nothing & - & $9(56 \%)$ & - & $8(36 \%)$ & $1(1 \%)$ & 3 & - & $93(82 \%)^{+}$ \\
\hline 4-week FU & $1(1 \%)$ & $1(11 \%)$ & $12(12 \%)$ & $4(18 \%)$ & $4(4 \%)$ & 1 & $1(20 \%)$ & $3(3 \%)$ \\
\hline 3-month FU & $34(33 \%)$ & $1(11 \%)$ & $67(69 \%)^{+}$ & $8(36 \%)$ & $20(19 \%)$ & 4 & $1(20 \%)$ & $3(3 \%)$ \\
\hline 6-month FU & $10(10 \%)$ & $2(22 \%)$ & $2(2 \%)$ & $1(5 \%)$ & $1(1 \%)$ & 1 & - & $8(7 \%)$ \\
\hline 24-month FU & $5(5 \%)$ & - & $1(1 \%)$ & - & $2(2 \%)$ & 1 & $1(20 \%)$ & $3(3 \%)$ \\
\hline 12,24 , and 36-month FU & $32(31 \%)^{+}$ & $2(22 \%)$ & $4(4 \%)$ & $1(5 \%)$ & $2(2 \%)$ & - & $1(20 \%)$ & $3(3 \%)$ \\
\hline Other modality & $12(12 \%)$ & $1(11 \%)$ & $4(4 \%)$ & - & $38(36 \%)^{+}$ & 1 & $1(20 \%)$ & $1(1 \%)$ \\
\hline Resection & $1(1 \%)$ & - & $3(3 \%)$ & - & $34(32 \%)^{+}$ & 2 & - & - \\
\hline Other & $8(8 \%)^{a}$ & - & $4(4 \%)^{b}$ & - & $3(3 \%)^{c}$ & 1 & - & - \\
\hline Overmanagement & $60(58 \%)$ & N/A & $26(27 \%)$ & N/A & - & N/A & N/A & $21(18 \%)$ \\
\hline Undermanagement & $6(6 \%)$ & N/A & $4(4 \%)$ & N/A & $33(31 \%)$ & N/A & N/A & - \\
\hline
\end{tabular}

Case 1 represents a persistent, pure ground-glass SSN $(15 \times 14 \mathrm{~mm})$; case 2 shows a new part-solid SSN with a small solid component (total lesion size $20 \times 16 \mathrm{~mm}$, solid component size $3 \times 4 \mathrm{~mm}$ ); case 3 shows a persistent part-solid SSN with a large solid component (total lesion size $25 \times 25 \mathrm{~mm}$, solid component size $12 \times 8 \mathrm{~mm})$; and case 4 presents a triangular solid nodule at a perifissural location $(10 \times 5 \mathrm{~mm})$

$\mathrm{FU}=$ follow-up $;^{*}=$ correct characterization of the pulmonary nodule; ${ }^{+}=$correct management strategy according to Fleischner recommendations for SSN management; ${ }^{a}=$ three respondents did not provide management choice; ${ }^{\mathrm{b}}=$ two respondents did not provide management choice; ${ }^{\mathrm{c}}=$ one respondent did not provide management choice

diameters in the axial plane, as described in the Fleischner document. Only $13 \%(28 / 141)$ of the pulmonologists who personally perform measurements using calipers $(58 \%, 141 / 243)$ did so in a manner that conformed.

\section{Discussion}

The present study evaluates the awareness of and conformance to the Fleischner recommendations for SSN

Table 3 Chosen management strategies per case by pulmonologists or pulmonology residents

\begin{tabular}{|c|c|c|c|c|c|c|c|c|}
\hline & \multicolumn{2}{|l|}{ Case 1} & \multicolumn{2}{|l|}{ Case 2} & \multicolumn{2}{|l|}{ Case 3} & \multicolumn{2}{|l|}{ Case 4} \\
\hline & SSN & No SSN & SSN & No SSN & SSN & No SSN & SSN & No SSN \\
\hline Management strategy & $224^{*}$ & 19 & $187^{*}$ & 56 & $180^{*}$ & 63 & 33 & $210^{*}$ \\
\hline Nothing & $2(1 \%)$ & $7(37 \%)$ & $1(1 \%)$ & $27(48 \%)$ & $1(1 \%)$ & $37(59 \%)$ & $3(9 \%)$ & $158(75 \%)^{+}$ \\
\hline 4-week FU & $21(9 \%)$ & $2(11 \%)$ & $41(22 \%)$ & $5(9 \%)$ & $10(6 \%)$ & $4(6 \%)$ & $2(6 \%)$ & $3(1 \%)$ \\
\hline 3-month FU & $94(42 \%)$ & - & $80(43 \%)^{+}$ & $11(20 \%)$ & $29(16 \%)$ & $2(3 \%)$ & $16(48 \%)$ & $20(10 \%)$ \\
\hline 6-month FU & $17(8 \%)$ & $3(16 \%)$ & $7(4 \%)$ & $4(7 \%)$ & $2(1 \%)$ & $2(3 \%)$ & $7(21 \%)$ & $20(10 \%)$ \\
\hline 24-month FU & $7(3 \%)$ & $2(11 \%)$ & $2(1 \%)$ & - & - & $2(3 \%)$ & $2(6 \%)$ & $4(2 \%)$ \\
\hline 12, 24 and 36-month FU & $26(12 \%)^{+}$ & $3(16 \%)$ & $9(5 \%)$ & $3(5 \%)$ & $5(3 \%)$ & $1(2 \%)$ & $2(6 \%)$ & $5(2 \%)$ \\
\hline Other modality & $38(17 \%)$ & $2(11 \%)$ & $35(19 \%)$ & $4(7 \%)$ & $97(54 \%)^{+}$ & $13(21 \%)$ & - & - \\
\hline Resection & $13(6 \%)$ & - & $7(4 \%)$ & $1(2 \%)$ & $29(16 \%)^{+}$ & $1(2 \%)$ & $1(3 \%)$ & - \\
\hline Other & $6(3 \%)^{a}$ & - & $5(3 \%)^{b}$ & $1(2 \%)^{\mathrm{c}}$ & $7(4 \%)^{d}$ & $1(2 \%)$ & - & - \\
\hline Overmanagement & $187(83 \%)$ & N/A & $96(51 \%)$ & N/A & - & N/A & $\mathrm{N} / \mathrm{A}$ & $52(25 \%)$ \\
\hline Undermanagement & $11(5 \%)$ & N/A & $11(6 \%)$ & N/A & $54(30 \%)$ & N/A & $\mathrm{N} / \mathrm{A}$ & - \\
\hline
\end{tabular}

Case 1 represents a persistent, pure ground-glass SSN (15x14 mm); case 2 shows a new part-solid SSN with a small solid component (total lesion size $20 \times 16 \mathrm{~mm}$, solid component size $3 \times 4 \mathrm{~mm}$ ); case 3 shows a persistent part-solid SSN with a large solid component (total lesion size $25 \times 25 \mathrm{~mm}$, solid component size $12 \times 8 \mathrm{~mm})$; and case 4 presents a triangular solid nodule with a perifissural location $(10 \times 5 \mathrm{~mm})$

$\mathrm{FU}=$ follow-up ${ }^{*}=$ correct characterization of the pulmonary nodule; ${ }^{+}=$correct management strategy according to Fleischner recommendations for SSN management; ${ }^{a}=$ two respondents did not provide management recommendation; ${ }^{\mathrm{b}}=$ one respondent did not provide management recommendation; ${ }^{\mathrm{c}}=$ one respondent did not provide management choice; ${ }^{\mathrm{d}}=$ four respondents did not provide management choice 
Table 4 Awareness of Fleischner recommendations for SSN management among respondents

\begin{tabular}{|c|c|c|c|c|c|c|}
\hline & \multicolumn{2}{|l|}{ Radiologists } & & \multicolumn{2}{|l|}{ Pulmonologists } & \\
\hline & Aware & Unaware & & Aware & Unaware & \\
\hline All respondents & $111 / 119(93 \%)$ & $8 / 119(7 \%)$ & & $169 / 243(70 \%)$ & $74 / 243(30 \%)$ & \\
\hline $\begin{array}{l}\text { Academic } \\
\text { Non-academic }\end{array}$ & $\begin{array}{l}63 / 67(94 \%) \\
48 / 52(92 \%)\end{array}$ & $\begin{array}{l}4 / 67(6 \%) \\
4 / 52(8 \%)\end{array}$ & $P=0.09$ & $\begin{array}{l}110 / 155(71 \%) \\
59 / 88(67 \%)\end{array}$ & $\begin{array}{l}45 / 155(29 \%) \\
29 / 88(33 \%)\end{array}$ & $P=0.52$ \\
\hline $\begin{array}{l}\text { Teaching practice } \\
\text { Non-teaching practice }\end{array}$ & $\begin{array}{l}97 / 102(95 \%) \\
14 / 17(82 \%)\end{array}$ & $\begin{array}{l}5 / 102(5 \%) \\
3 / 17(18 \%)\end{array}$ & $P=0.09$ & $\begin{array}{l}129 / 182(71 \%) \\
40 / 61(66 \%)\end{array}$ & $\begin{array}{l}53 / 182(29 \%) \\
21 / 61(34 \%)\end{array}$ & $P=0.44$ \\
\hline $\begin{array}{l}\text { Less experienced ( }<5 \text { years }) \\
\text { More experienced ( } 5 \text { or more })\end{array}$ & $\begin{array}{l}17 / 21(81 \%) \\
94 / 98(96 \%)\end{array}$ & $\begin{array}{l}4 / 21(19 \%) \\
4 / 98(4 \%)\end{array}$ & $P=0.03$ & $\begin{array}{l}43 / 68(63 \%) \\
126 / 175(72 \%)\end{array}$ & $\begin{array}{l}25 / 68(37 \%) \\
49 / 175(28 \%)\end{array}$ & $P=0.18$ \\
\hline $\begin{array}{l}\text { Smaller practice }(<10 \text { specialists }) \\
\text { Larger practice }(10 \text { or more })\end{array}$ & $\begin{array}{l}30 / 31(97 \%) \\
81 / 88(92 \%)\end{array}$ & $\begin{array}{l}1 / 31(3 \%) \\
7 / 88(8 \%)\end{array}$ & $P=0.68$ & $\begin{array}{l}112 / 155(72 \%) \\
57 / 88(65 \%)\end{array}$ & $\begin{array}{l}43 / 155(28 \%) \\
31 / 88(35 \%)\end{array}$ & $P=0.22$ \\
\hline Dept. without chest fellowship-trained specialist & 29/31 (94\%) & $2 / 31(6 \%)$ & $P=1.00$ & N/A & & \\
\hline Dept. with at least 1 chest-fellowship specialist & $82 / 88(93 \%)$ & $6 / 88(7 \%)$ & & & & \\
\hline $\begin{array}{l}\text { Number SSNs encountered ( } 10 \text { or less } \mathrm{p} / \mathrm{y}) \\
\text { Number SSNs encountered ( }>10 \mathrm{p} / \mathrm{y})\end{array}$ & $\begin{array}{l}16 / 20(80 \%) \\
95 / 99(96 \%)\end{array}$ & $\begin{array}{l}4 / 20(20 \%) \\
4 / 99(4 \%)\end{array}$ & $P=0.03$ & $\begin{array}{l}68 / 113(60 \%) \\
101 / 130(78 \%)\end{array}$ & $\begin{array}{l}45 / 113(40 \%) \\
29 / 130(22 \%)\end{array}$ & $P<0.01$ \\
\hline
\end{tabular}

Dept. $=$ Department; SSN = Subsolid pulmonary nodule

management among radiologists and pulmonologists in clinical practice. Our survey shows that over two years after publication, awareness is widespread. Nevertheless, conformance to the FR varies considerably and overmanagement with more follow-up or additional diagnostic procedures not indicated is common.

To our knowledge, this is the first study to evaluate the awareness of and conformance to the Fleischner recommendations for SSN management. Previously, a number of survey studies have been performed to evaluate management of small, solid pulmonary nodules [11-15]. A shared conclusion of these studies was that there is high heterogeneity among specialists with respect to guideline conformance. Contrarily, a high percentage of respondents reported awareness of the guidelines [11]. In our study, which included both radiologists and pulmonologists, the results are comparable. Despite high awareness and straightforward clinical scenarios, between 31 and $69 \%$ of the radiologists chose management recommendations not corresponding to the FR. Among pulmonologists, awareness was significantly lower and management of SSNs even more variable, which may well be due to the radiological origin of the recommendations. Most interestingly, we also observed a variable conformance to the FR within individual readers for the four lesions types.

There may be several explanations for management deviations: (1) respondents may be aware of the FR but apply them erroneously, use a deviating, locally developed protocol or no clearly described method at all. It remains unclear to which extent one or more of these factors contributed to the variability we observed. Besides the Fleischner recommendations for SSN management, other management standards are publicly available, with LUNG-RADS or ACCP guidelines being the most important [16, 17]. Interestingly however, among our respondents only a few individuals $(\mathrm{N}=7)$ reported to apply these guidelines in daily practice. (2) Respondents may not agree with the FR and therefore deliberately chose a deviating strategy. After all, the FR are mainly based on expert opinion due to limited availability of data, especially in a non-lung cancer screening setting. In our study, non-conformance was surprisingly mostly categorized as overmanagement. Therefore, disagreement due to the conservativeness of the FR does not seem to be a major explanatory factor for the heterogeneity. (3) Some deviation in management might be erroneously due to respondents who overread or ignored the presented clinical scenarios (e.g., new versus persistent SSN). This may at least partly explain why overmanagement was highest for the persistent, pure ground-glass nodule, with the description 'persistent' as the clue for yearly serial follow-up and not a 3-month follow-up scan.

\section{Awareness and conformance}

Awareness of the Fleischner recommendations for SSN management was significantly higher in clinicians with higher exposure to SSNs, and in more experienced radiologists. However, it has to be noted that awareness among radiologists was generally very high (ie. only eight individuals reported unawareness), limiting statistical power. Guideline awareness among pulmonologists - which was significantly lower and only showed association to SSN exposure-has not been assessed previously. Previously reported factors associated with guideline conformance in solid nodule management included awareness of the guidelines, presence of a written management policy, working in a teaching practice, fellowship training in cardiothoracic radiology (either personally or a direct colleague), and years of experience [11, 13, 14]. Our 


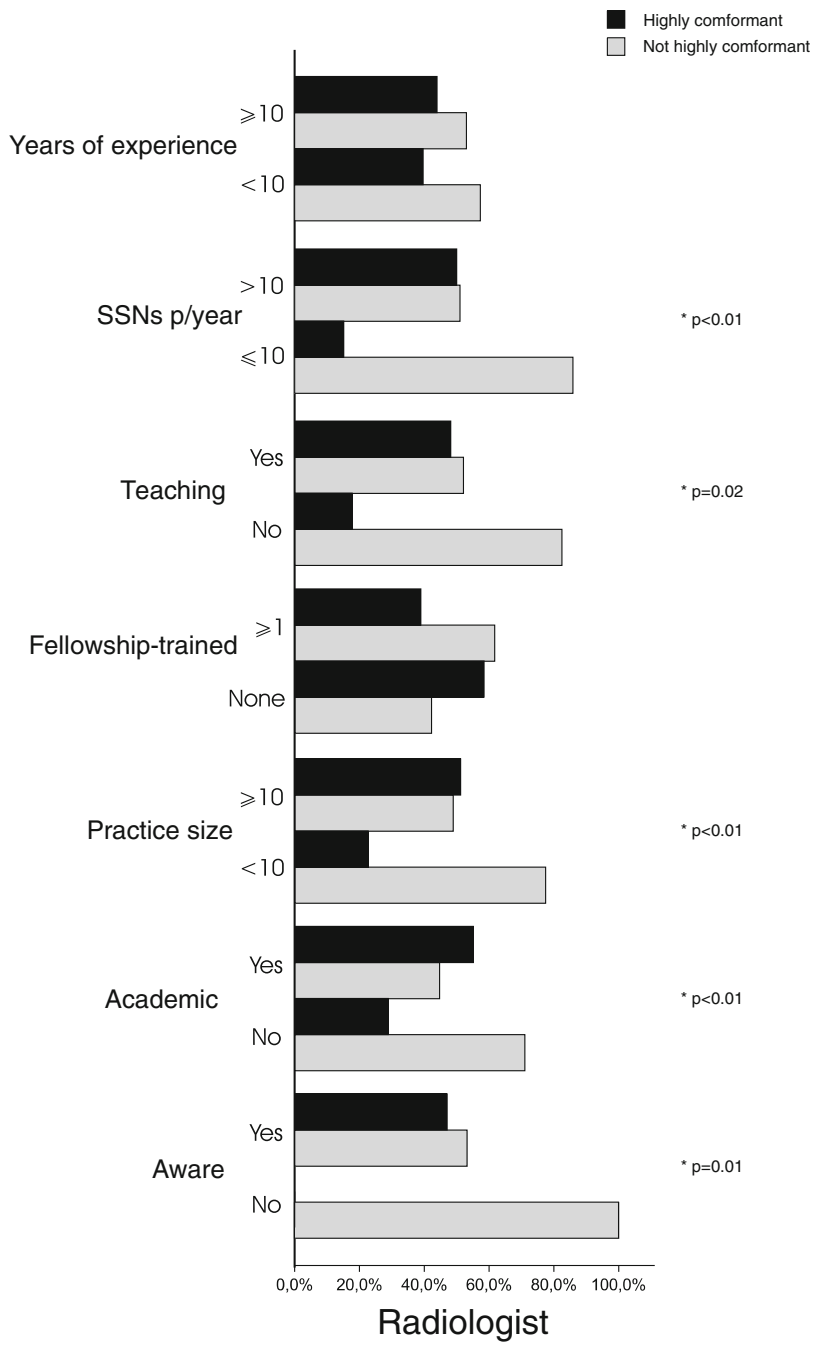

Fig. 3 Radiologists and pulmonologists background characteristics in association with high conformance to the Fleischner recommendations for SSN management. The figure shows the percentage of radiologists and pulmonologists that showed high conformity (correct management strategy in three or four out of four cases). In radiologists, high

results are largely comparable, with the exception that we did not find an influence of experience or fellowship-training in radiologists. This might be due to the fact that SSNs and their management are a relatively new entity, in which the more experienced clinicians have not yet developed their own long-standing practice patterns in solid nodule management [11]. Adding to what was previously discussed in the literature, we showed that higher exposure is associated with conformance.

\section{Over- and undermanagement}

The surprisingly strong tendency towards overmanagement is an important finding because unnecessary scans and invasive procedures lead to extra costs, radiation exposure, and potentially, to procedure-related risks. On the other hand, undermanagement is also a very important issue. It may lead

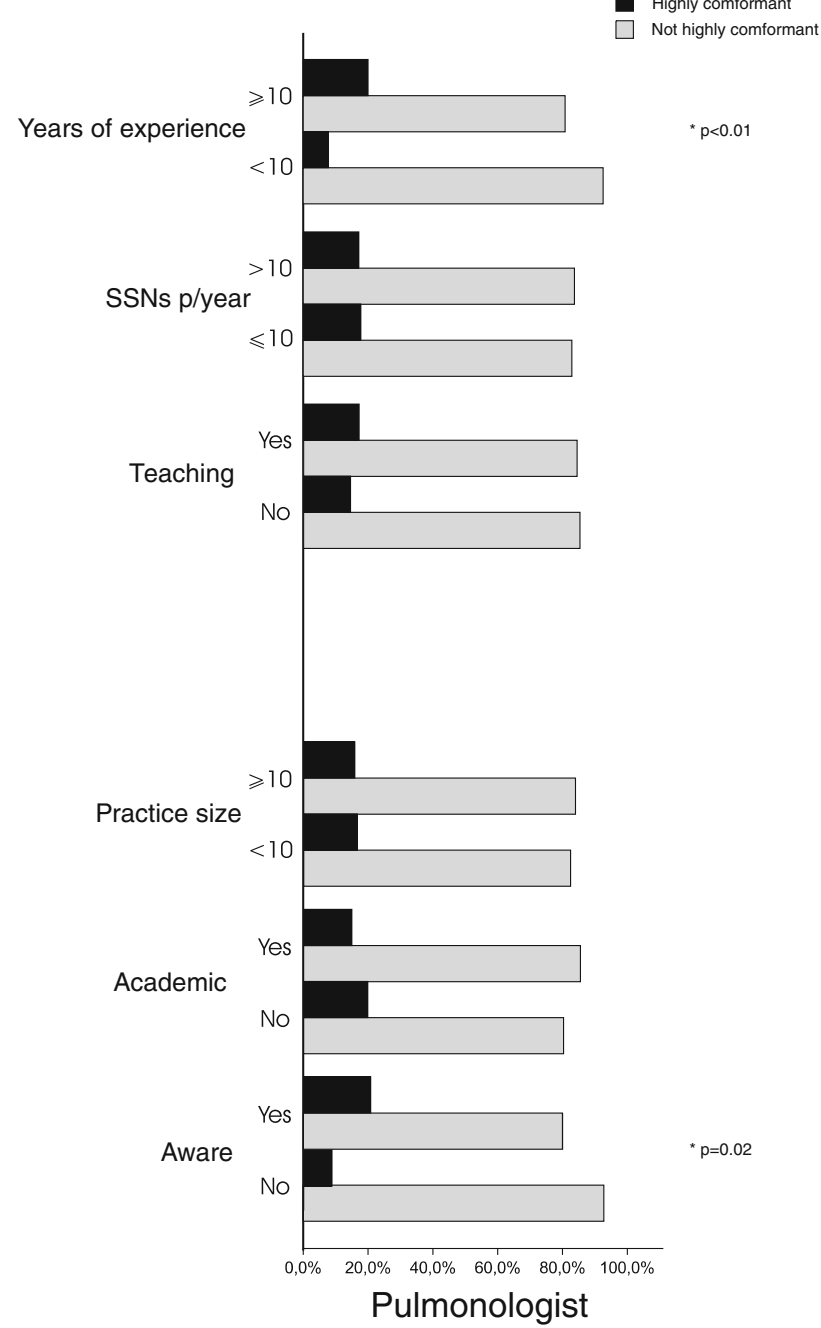

conformance was significantly associated with SSN exposure, teaching residents, working in a larger practice ( $\geq 10$ specialists), working in an academic centre, and awareness of the FR. In pulmonologists, high conformity was associated with more experience ( $\geq 10$ years) and awareness of the FR

to unnecessary morbidity and mortality caused by advanced tumour stages. The latter, however, is still under discussion and more results from long-term outcome studies are needed, given the mainly indolent and slowly progressing nature of SSNs.

We found that overmanagement according to the FR was common among both radiologists and pulmonologists (18$58 \%$ and $25-83 \%$, respectively), which is in line with results from the previous studies in solid nodule management, which reported overmanagement in 39-62 \% [11] and 17$93 \%$ [15]. Pulmonologists showed a stronger tendency towards overmanagement compared to radiologists, including invasive procedures as biopsy and resection. The reason for this remains unclear based on our results, but might be related to their reported lack of confidence in SSN management, with tissue diagnosis and a definite answer as the safest option. 
On the other hand, we found undermanagement for the persistent part-solid subsolid lesion by both groups of respondents. This type of lesion, if persistent and demonstrating a solid component exceeding $5 \mathrm{~mm}$, is considered highly suggestive for invasive adenocarcinoma. Nevertheless, $33 \%$ of radiologists and $54 \%$ of pulmonologists would have not recommended an immediate (invasive) work-up. This indicates either insufficient knowledge about the meaning of this type of lesion or the desire to avoid overdiagnosis by at least a subgroup of colleagues.

As mentioned above, some deviation in management may be erroneously due to respondents overlooking or ignoring clinical information on nodule persistence. This seems most relevant in case 1 , in which 33 and $42 \%$ of the radiologists and pulmonologists, respectively, called for a 3-month followup interval (which is indicated in the case of a new nodule) instead of the indicated serial annual follow-up (in the case of a persistent SSN). To a lesser extent, this might also account for some of the undermanagement in case 3, in which 19 and $16 \%$ of the respondents chose a 3-month follow-up interval instead of further work-up. Determining the true impact of respondents noting persistence in the presented cases is impossible, but over- and undermanagement may thus be somewhat lower than presented. Nevertheless, this argument cannot fully explain the large heterogeneity throughout and between the cases and respondents. Moreover, previous guideline conformance studies showed comparable percentages of non-conformance. We believe that the heterogeneity in SSN management found in this study is mainly caused by personal interpretation and an inherent tendency to adjust guidelines by individual clinicians.

\section{Measurements of nodule dimensions}

We further found that only a small minority of respondents obtain transverse measurements in two dimensions, as proposed in the Fleischner document. Most indicated that they measure the single maximum nodule diameter in either the axial or any other plane. In our study the influence of our finding was likely limited given the small number of nodules and a solid component size clearly under or over the threshold of $5 \mathrm{~mm}$ (case 2 and 3). Also, the influence of our finding in general is probably limited, given that one should only determine whether the SSN or its solid component is $\leq 5 \mathrm{~mm}$ or $>5 \mathrm{~mm}$. On the contrary, for the evaluation of solid pulmonary nodules where a similar measurement technique should be used and classification into four different size categories is required $(<4 \mathrm{~mm}, 4-6 \mathrm{~mm}, 6-8 \mathrm{~mm}$ and $<8 \mathrm{~mm}$; [18]), this may well be of far more importance.

The strength of the current study is that we succeeded in including a sufficient number of both radiologists and pulmonologists. Given that in clinical practice detection and follow-up of subsolid pulmonary nodules is not a monodisciplinary affair, it is important to obtain information on SSN management in both groups involved. Also, we were able to include both single-slice and animated multi-slice images of the nodules in the online questionnaire. We therefore also tested knowledge on nodule interpretation, which more closely resembles clinical practice than a written clinical scenario, as previously used in other survey studies on solid nodule management.

Our study also has some limitations. First, as with any survey there might be a response bias, which can neither be excluded nor quantified. However, in case a response bias was introduced, we believe it is far more likely that there has been weighting towards respondents with an affinity for subsolid nodules than for those without. If so, our results on awareness and conformance are probably higher than in reality. Second, due to technical reasons we only included solitary SSNs in the survey, while the FR also includes management of multiple subsolid nodules. Since management recommendations between solitary and multiple SSNs do not differ significantly and are both part of the same Fleischner document, we believe our study design is nevertheless valid to assess the presented study purpose.

In conclusion, although awareness of the Fleischner recommendations for SSN management is widespread among both radiologists and pulmonologists, management choices differ substantially from these recommendations, and over- and undermanagement are common.

Acknowledgments We would like to thank Prof. Dr. E.H.D. Bel and Dr. T. Franquet, presidents of the European Respiratory Society (ERS) and European Society of Thoracic Imaging (ESTI), respectively, for their willingness and effort to send out the survey to their members.

The scientific guarantor of this publication is CM Schaefer-Prokop. The authors of this manuscript declare no relationships with any companies, whose products or services may be related to the subject matter of the article. This study was financially supported by the Dutch Cancer Foundation, project KUN-2013-6110. No complex statistical methods were necessary for this paper. Institutional Review Board approval was obtained. Methodology: prospective, multicenter study.

Open Access This article is distributed under the terms of the Creative Commons Attribution-NonCommercial 4.0 International License (http:// creativecommons.org/licenses/by-nc/4.0/), which permits any noncommercial use, distribution, and reproduction in any medium, provided you give appropriate credit to the original author(s) and the source, provide a link to the Creative Commons license, and indicate if changes were made.

\section{References}

1. Henschke CI, Yankelevitz DF, Mirtcheva R, Mcguinness G, McCauley D, Miettinen OS (2002) CT screening for lung cancer: frequency and significance of part-solid and nonsolid nodules. AJR Am J Roentgenol 178:1053-1057 
2. Henschke CI, Yankelevitz DF, Yip R et al (2012) Lung cancers diagnosed at annual CT screening: volume doubling times. Radiology 263:578-583

3. Fang W, Xiang Y, Zhong C, Chen Q (2014) The IASLC/ATS/ERS classification of lung adenocarcinoma-a surgical point of view. $\mathrm{J}$ Thorac Dis 6:S552-S560

4. Noguchi M, Morikawa A, Kawasaki M et al (1995) Small adenocarcinoma of the lung. Histologic characteristics and prognosis. Cancer 75:2844-2852

5. Travis WD, Brambilla E, Noguchi M et al (2011) IASLC/ATS/ERS international multidisciplinary classification of lung adenocarcinoma. J Thorac Oncol 6:244-285

6. Godoy MC, Naidich DP (2012) Overview and strategic management of subsolid pulmonary nodules. J Thorac Imaging 27: 240-248

7. Goo JM, Park CM, Lee HJ (2011) Ground-glass nodules on chest $\mathrm{CT}$ as imaging biomarkers in the management of lung adenocarcinoma. AJR Am J Roentgenol 196:533-543

8. Naidich DP, Bankier AA, Macmahon H et al (2013) Recommendations for the management of subsolid pulmonary nodules detected at CT: a statement from the Fleischner Society. Radiology 266:304-317

9. Top-10 most-viewed Radiology articles online. Available from http://pubs.rsna.org/journal/radiology - Last accessed November 2015

10. de Hoop B, van Ginneken B, Gietema H, Prokop M (2012) Pulmonary perifissural nodules on CT scans: rapid growth is not a predictor of malignancy. Radiology 265(2):611-616
11. Eisenberg RL, Bankier AA, Boiselle PM (2010) Compliance with Fleischner Society guidelines for management of small lung nodules: a survey of 834 radiologists. Radiology 255:218-224

12. Quint LE, Watcharotone K, Myles JD, Gruppen LD, Mullan PB (2011) Incidental findings at chest CT: a needs assessment survey of radiologists' knowledge. Acad Radiol 18:1500-1506

13. Prosch H, Strasser G, Oschatz E, Schober E, Schneider B, Mostbeck GH (2006) Management of patients with small pulmonary nodules: a survey of radiologists, pulmonologists, and thoracic surgeons. AJR Am J Roentgenol 187:143-148

14. Jeudy J, White CS, Munden RF, Boiselle PM (2008) Management of small (3-5-mm) pulmonary nodules at chest CT: global survey of thoracic radiologists. Radiology 247:847-853

15. Esmaili A, Munden RF, Mohammed TL (2011) Small pulmonary nodule management: a survey of the members of the Society of Thoracic Radiology with comparison to the Fleischner Society guidelines. J Thorac Imaging 26:27-31

16. American College of Radiology. Lung CT Screening Reporting and Data System (Lung-RADS). Last accessed at http://www.acr.org/ Quality-Safety/Resources/LungRADS in November 2015

17. Gould MK, Donington J, Lynch WR et al (2013) Evaluation of individuals with pulmonary nodules: when is it lung cancer? Diagnosis and management of lung cancer, 3rd ed: American College of Chest Physicians evidence-based clinical practice guidelines. Chest 143:e93S-120S

18. Macmahon H, Austin JH, Gamsu G et al (2005) Guidelines for management of small pulmonary nodules detected on CT scans: a statement from the Fleischner Society. Radiology 237:395-400 